\title{
On the Formation and Realization of the Value of Traditional Chinese Medicine Health Tourism Resources
}

\author{
Yawen Liao, Qin Huang, Yang He, Peiqian Wang, Li Wang \\ Jiangxi University of Traditional Chinese Medicine \\ Nanchang 330004
}

\begin{abstract}
Traditional Chinese medicine health tourism is a special way of tourism and its formation and realization of value has a unique mechanism. The paper analyzes the value system of traditional Chinese medicine tourism resources from the perspectives of Management, Marketing, and Economics, etc., and concludes that the value of traditional Chinese medicine health tourism is formed under dynamic mechanism, coordination mechanism and coupling mechanism, and is realized through cooperation mechanism, element mechanism and cognitive mechanism.
\end{abstract}

Keywords-Traditional Chinese medicine; Tourism value; Formation mechanism; Realization mode

\section{INTRODUCTION}

In our country, traditional Chinese medicine culture has farreaching influence on our daily life. The trend of healthcare through traditional Chinese medicine lasts for a long time. If we can combine the traditional Chinese medicine culture over 3,000 years with tourism industry, it can not only strengthen people's understanding of traditional Chinese medicine, meet people's demands for health maintenance, health care, tourism and other needs, but also can promote the traditional Chinese medicine culture.

According to the prediction of World Health Organization, service industry related to medical health and sightseeing leisure tourism will occupy top two of the global industry by 2020 , and the combined output value will account for $22 \%$ of the global GDP. In 2016, the Guiding Opinions on Promoting the Development of Traditional Chinese Medicine Health Tourism jointly issued by China National Tourism Administration and State Administration of Traditional Chinese Medicine also mentioned that the number of traditional Chinese medicine health tourism will reach $3 \%$ of the total number of tourists by 2020, and the output will be up to RMB 300 billion; By 2025, this data will rise to $5 \%$ and RMB 500 billion [1]. The economic development brought by the reform and people's demand for spiritual consumption has greatly inspired the new demands of health tourism. Traditional Chinese medicine Tourism industry is facing unprecedented opportunities for development.

\section{THE CONNOTATION AND COMPOSITION OF THE VALUE of Traditional CHINESE MEdicine HeAlth TOURISM RESOURCES}

Traditional Chinese medicine health tourism is a tourism with characteristics that has developed rapidly and is becoming more and more popular in recent years. Currently, it is authoritatively defined as a tourism activity that relies on traditional Chinese medicine resources, for the purpose of promoting health, to carry out health maintenance, experience, leisure, sightseeing and vacation, etc., and also inherit and carry forward the traditional Chinese medicine culture and experience traditional Chinese medicine service [2]. Previous research in China made some thought about traditional Chinese medicine tourism resources but did not make in-depth discussion about the value of traditional Chinese medicine tourism resources. The author holds that the value of traditional Chinese medicine health tourism resource refers to the degree of attraction to tourists, which can inspire the tourist's tourism motivation, meet the tourist's experience demands for health care and relaxing the mind and body, and thus bring a series of economic and social benefits.

Tourism value system is composed of tourism value subject, tourism value object and tourism value realization medium [3]. Traditional Chinese medicine tourism resources and tourist are two main parts of tourism value system of traditional Chinese medicine, and there is also an indispensable part between the two parts - tourism value realization path and platform. From the characteristics of traditional Chinese medicine health tourism resources, in addition to the appreciation value, economic value, public benefit value and scientific research value of common tourism mode, it also has the health care value that other tourism resources cannot be satisfied. This is the most distinctive feature of traditional Chinese medicine health tourism and is also the most attractive part for tourists. 
cooperation between the upstream and downstream, namely, the transmission of "smile curve", the whole Chinese medicine health tourism industry value chain gets supported finally.

Coupling Mechanism Traditional Chinese medicine health tourism is an innovative tourism model closely combining health and tourism through the medium of traditional Chinese medicine. The traditional Chinese medicine treatment techniques, such as acupuncture, scrapping, cupping, traditional Chinese medicine exercises and other health-care activities, are matched with the form of green scenery and visiting experience in the eco-tourism, which not only contains the value of the traditional Chinese medicine therapy but also retains the value of eco-tourism, thus achieving a value of $1+1>2$ sublimation. Tourism activity is the carrier of culture, culture is the soul of tourism, tourism industry and cultural industry can achieve complementarities and win-win, and there exists natural coupling between them. The gradual coupling of traditional Chinese medicine culture with tourism resource, tourism capital and tourism market from the primary to the advanced stage will play the combined function of culture and tourism, form a traditional Chinese medicine culture tour with characteristics, and can effectively improve the quality value of tourism resources [7].

Through the analysis of the above mechanisms, it can be concluded the basic model of the value of traditional Chinese medicine health tourism resource: The demand for healthcare drives the tourist to produce the motive force for traditional Chinese medicine health tourism. The relevant subjects of the traditional Chinese medicine health tourism industry build a platform for realizing the demand for healthcare through collaboration and cooperation, provides a tourism mode with multiple functions, such as "Health + Tourism" and "Traditional Chinese Medicine Culture + Tourism", and finally achieves the polymerization of the value of the traditional Chinese medicine health tourism resource.

\section{THE REALIZATION MECHANiSM OF THE VALUE OF Traditional Chinese Medicine HeAlth TOURISM RESOURCES}

The value of the resource itself is a prerequisite for the realization of the value. In addition, the ultimate realization of the value of traditional Chinese medicine health tourism resources also needs the combination of each subject and element in relevant with traditional Chinese medicine health tourism industry and takes effective mechanism for guidance.

\section{A. Cooperation Mechanisms}

Tourism Enterprise Tourism enterprise is one of the main subjects that promotes the realization of the value of traditional Chinese medicine health tourism resources and plays a core and direct role through scalization and market operations. Under the function of market competition mechanism, it can promote the tourism enterprise to optimize the efficiency of utilizing the resources in creative planning, content production, product positioning, marketing, etc., provide high-quality tourism service, and play the value of traditional Chinese medicine health tourism. 
Healthcare and Pension Service Agencies The healthcare agencies and the traditional Chinese medical service agencies use their own understanding and research advantages of traditional Chinese medicine to provide professional technical operation and auxiliary explanation, organize the technical personnel of traditional Chinese medicine to provide the tourism enterprises with products and services corresponding to traditional Chinese medicine tourism, and coordinate with the tourism enterprises to construct a modern innovation management system that is suitable for the characteristics of the traditional Chinese medicine health tourism, so as to inject the economic value and the health care value into traditional Chinese medicine health tourism. At the same time, it is also necessary to mobilize the pension and community service agencies to participate in traditional Chinese medicine health tourism, which can not only provide the corresponding consumption subject for traditional Chinese medicine health tourism, but also can purchase professional services and products from them, expand their own scope of service, improve the service quality, and achieve the effect of mutual benefit and win-win.

Government The realization of the value of traditional Chinese medicine health tourism cannot be separated from a good social environment created by the government. At present, the government still serves as resource developer and tourism manager in the tourism market, and excessive administrative intervention has affected the market environment of fair competition in the tourism industry. Therefore, the government should change its role from a "paddler" to a "steersman", and focus on the formulation and supervision of laws and regulations of traditional Chinese medicine tourism, so as to standardize the behavior of traditional Chinese medicine tourism market service from the system.

Within the entire cooperation system, the government should lead the cooperation in relevant agencies, formulate the overall development plan and laws and regulations corresponding to Chinese traditional Chinese medicine health tourism, encourage the tourism enterprises to actively contact the healthcare service agencies, exert their own advantages, and join forces to implement the development of tourism projects. The healthcare agencies and the traditional Chinese medical service agencies should develop multiple kinds of traditional Chinese medicine services and products, and promote them to the masses through the platforms of tourism enterprises, pension agencies and community service agencies, so as to attract them to experience traditional Chinese medicine health tourism. At the same time, these subjects should also encourage each other to guarantee the accumulation of funds in the process of realizing the value of traditional Chinese medicine health tourism resources, the application of the development management, the medical talents and the technical elements in the realization process of the value of traditional Chinese medicine health tourism resources, and the realization of value of traditional Chinese medicine health tourism resources in a good institutional environment.

\section{B. Element Mechanism}

Fund Element Fund is an essential element in realizing the value of traditional Chinese medicine health tourism resources. To play the role of investment, it is necessary to introduce the market mechanism to create a fair market competition environment, and finally form a diversified pattern combining government investment and social investment. From a macro perspective, it includes the investment policy orientation, investment structure, layout, scale, mode, mechanism, method and subject, as well as the investment efficiency that promotes the development of Chinese medicine tourism. From a micro perspective, it mainly involves the research on the tourism development subject to invest in specific tourism development projects, including a comprehensive study of the utilization of the tourism investment policy, the tourism industry, the feasibility of tourism development project, the investment portfolio and the investment risk, as well as the influence of tourism law on tourism investment [8].

Talent and Technology Element The talent and technology element is the core element for the realization of the value of traditional Chinese medicine health tourism resources, which determines the degree of realization to a certain extent. The realization of the value of traditional Chinese medicine health tourism resources needs to include the professional technical talents of traditional Chinese medicine (eg. professional doctors of traditional Chinese medicine, traditional Chinese medicine technicians, etc.), traditional Chinese medicine tourism industry management talents, traditional Chinese medicine explanation and promotion talents, etc. In the technical aspects, it is important to follow the trend of the times, adopt the mode of "Internet + Traditional Chinese Medicine", such as the $\mathrm{O} 2 \mathrm{O}$ mode of food, specialty and medicated diet, the development and research of traditional Chinese medicine health tourism mobile game (mobile handheld game), research and development of $360^{\circ}$ panoramic display platform APP of Pharm China, B2C online appointment of Chinese medicine health maintenance and healthcare projects, etc [9].

System Elements The system has direct influence on the realization of the value of tourism resources, such as contract mode and behavior, enterprise integration and merger, utilization and design of assets specificity, division in industrial cluster, internalization of the external problems, etc. All these problems restrict the realization degree of the value of traditional Chinese medicine health tourism resources. Although corresponding policies have been launched from the State to local places to guide the development of traditional Chinese medicine health tourism, and a number of supported traditional Chinese medicine tourism collaborative innovation centers have been established, the actual development system implemented still needs to be refined and perfected so as to improve the utilization efficiency of the traditional Chinese medicine health tourism resources to the maximum.

Cognitive mechanism Tourist's tourism behavior and tourism consumption are the ultimate realization of the value of traditional Chinese medicine health tourism resources. Cognitive mechanisms have major impacts on the generation of 
behavior. Tourists selectively save the perceptions that they are willing to remember and then have the willingness to pay in the future, that is, the cognitive filtering mechanism. In addition, the tourism activities of tourists can be divided into three parts: pre-tourism, while-tourism, and post-tourism. Therefore, they are corresponding to pre-tourism cognition, while-tourism cognition, and post-tourism cognition respectively. The three parts influence and interact with each other to form a circulation mechanism [10]. According to the analysis of cognitive mechanism, the value of traditional Chinese medicine health tourism should take into account the preferences of consumers in the process and should care for the people. Since traditional Chinese medicine health tourism has not yet been popularized, it is necessary to strengthen the propaganda of traditional Chinese medicine tourism, provide tourists with scientific, authentic and straightway introductions of traditional Chinese medicine and tourist areas, and use the cognitive circulation mechanism to attract and retain the tourists. In this way, the value of traditional Chinese medicine health tourism resources can be effectively realized.

Through the above analysis, we can get such a value realization model of the traditional Chinese medicine health tourism resource: The tourism enterprises, healthcare, pension agencies and governments, as the main subjects of traditional Chinese medicine health tourism industry, encourage and cooperate with each other to provide high-quality traditional Chinese medicine health tourism products under the combined function of the internal and external factors such as funds, technology, talents and systems. The cognition of tourists is constantly selected and circulated, and then reflected in the choice of behavior, so as to complete the traditional Chinese medicine health tourism consumption, so that the actual benefits of traditional Chinese medicine health tourism resources can be finally generated and the due value can be realized.

\section{CONCLUSION}

According to the value formation and realization mechanism of traditional Chinese medicine health tourism resources, the development of traditional Chinese medicine health tourism industry should strengthen the collaboration between government, tourism enterprises and relevant service agencies, converge the activity of each element, and integrate economics, management science, psychology and other discipline thinking, so as to explore a scientific and effective path for the development of traditional Chinese medicine health tourism resources and contribute to the realization of the grand goal of health China.

\section{ACKNOWLEDGMENT}

Fund Project: Scientific Research Project of Traditional Chinese Medicine of Health and Family planning Commission of Jiangxi Province: Research on the Value Formation and Realization Mechanism of Traditional Chinese Medicine Health Tourism Resources, Project: 2016A037.

\section{REFERENCES}

[1] China National Tourism Administration and State Administration of Traditional Chinese Medicine. Guiding opinions on promoting the development of traditional Chinese medicine health tourism[ N]. Journal of Traditional Chinese Medicine, 2015-11-26 (003).

[2] Guangqin Hu, Guoming Pang, Yanfen She, Huiping Su, Honggang Xu, Mingchi Luo, Yanbo Yao, Jianchao Xi, Shuguang Yu, Jun Ma, Lidong Zhao, Zhiwei Wang, and Xiaoyong Chen. Study on the classification and evaluation standard of health tourism in traditional Chinese medicine [J]. World Chinese Medicine, 2017, 12 (05): 1191-1199.

[3] Xiaoping Hu. Composition and Characteristics of Tourism Value[N]. China Tourism News, 2008-04-16 (011).

[4] Xuke Wang. The theoretical and empirical research on the driving mechanism of urban tourism development[M]. Beijing: Economic Science Press, 2010.

[5] Hua Peng. An analysis of driving mechanism and driving force model of tourism development [J] Tourism, 1999, 14 (6): 39-44.

[6] Chaozhi Zhang, Zeng Deng, and Wang You. An analysis of the tourism industrial value chain based on the perspective of tourism experience [J]. Tourism Tribune, 2010, 25 (06): 19-25.

[7] Fengxia Li and Yanying Cao. Coupling development theory and empirical analysis in cultural industry and tourism industry --Taking Shandong Province as an example[J]. Journal of Hubei University of Economics, 2017, 15 (02): 53-59.

[8] Chunyan Zhang. Study on formation and realization mechanism of ice and snow tourism resource value [D]. Harbin Institute of Technology, 2008.

[9] Kai Huang, Shuangyan Yu, Feifei Shang, Xue Ji, and Han Sun. Internet promotion study report of traditional Chinese medicine health tourism in Zhangshu City [J]. Tourism Overview (The 2nd half of the Month), 2017 (01): 15-16.

[10] Guang Li and Yanling Li. Study on realization of the ice and snow sport tourism resources in mid-south region of china $[\mathrm{J}]$. Chinese Sports Science and Technology, 2015, 51 (04): 117-124. 